\title{
Fredholm-type integral equation in controlled metric-like spaces
}

\author{
Wasfi Shatanawi ${ }^{1,2}$, Nabil Mlaiki $^{1^{*}} \mathbb{D}$, Doaa Rizk ${ }^{3}$ and Enyinda Onunwor ${ }^{4}$
}

\section{"Correspondence:}

nmlaiki@psu.edu.sa;

nmlaiki2012@gmail.com

1 Department of Mathematics and

General Sciences, Prince Sultan

University, Riyadh, 11586, Saudi

Arabia

Full list of author information is

available at the end of the article

\begin{abstract}
In this article we make an improvement in the Banach contraction using a controlled function in controlled metric like spaces, which generalizes many results in the literature. Moreover, we present an application on Fredholm type integral equation.
\end{abstract}

Keywords: Fixed point; Controlled metric-like spaces; Fredholm-type integral equations

\section{Introduction}

One of the most interesting applications of fixed point theory is solving integral and differential equations; see, for example, [1]. The Banach contraction principle was generalized many times to extend its application. As an example of these generalizations, $b$-spaces (see [2]) are an extension of the regular metric spaces; see [3-15]. Lately, Kamran [16] introduced what the so-called extended $b$-metric spaces by adding a control function $\theta(\mathfrak{p}, \mathfrak{q})$ in the triangle inequality. For more on $b$-metric spaces and its extensions, we refer the reader to [17-23]. First, we start by reminding the reader the definition of extended $b$ metric spaces.

Definition 1.1 ([16]) Consider the set $X \neq \emptyset$ and $\theta: X \times X \rightarrow[1, \infty)$. Let $d_{e}: X \times X \rightarrow$ $[0, \infty)$ be such that for all $\mathfrak{p}, \mathfrak{q}, z \in X$,

(1) $d_{e}(\mathfrak{p}, \mathfrak{q})=0$ if and only if $\mathfrak{p}=\mathfrak{q}$;

(2) $d_{e}(\mathfrak{p}, \mathfrak{q})=d_{e}(\mathfrak{q}, \mathfrak{p})$;

(3) $d_{e}(\mathfrak{p}, \mathfrak{q}) \leq \theta(\mathfrak{p}, \mathfrak{q})\left[d_{e}(\mathfrak{p}, z)+d_{e}(z, \mathfrak{q})\right]$.

Then $\left(X, d_{e}\right)$ is called an extended $b$-metric space.

Mlaiki et al. [24] gave an extension to this type of metric spaces as follows.

Definition $1.2([24])$ Consider the set $X \neq \emptyset$ and $\varrho: X \times X \rightarrow[1, \infty)$. Suppose that a function $d_{c}: X \times X \rightarrow[0, \infty)$ satisfies the following:

(1) $d_{c}(\mathfrak{p}, \mathfrak{q})=0$ if and only if $\mathfrak{p}=\mathfrak{q}$;

(2) $d_{c}(\mathfrak{p}, \mathfrak{q})=d_{c}(\mathfrak{q}, \mathfrak{p})$;

(3) $d_{c}(\mathfrak{p}, \mathfrak{q}) \leq \varrho(\mathfrak{p}, z) d_{c}(\mathfrak{p}, z)+\varrho(z, \mathfrak{q}) d_{c}(z, \mathfrak{q})$ for all $\mathfrak{p}, \mathfrak{q}, z \in X$.

Then $\left(X, d_{c}\right)$ is called a controlled metric-type space.

(c) The Author(s) 2021. This article is licensed under a Creative Commons Attribution 4.0 International License, which permits use, sharing, adaptation, distribution and reproduction in any medium or format, as long as you give appropriate credit to the original author(s) and the source, provide a link to the Creative Commons licence, and indicate if changes were made. The images or other third party material in this article are included in the article's Creative Commons licence, unless indicated otherwise in a credit line to the material. If material is not included in the article's Creative Commons licence and your intended use is not permitted by statutory regulation or exceeds the permitted use, you will need to obtain permission directly from the copyright holder. To view a copy of this licence, visit http://creativecommons.org/licenses/by/4.0/. 
In 2021, a new generalization of the $b$-metric spaces introduced in [25], the so-called controlled metric-like spaces.

Definition $1.3([25])$ Consider the set $X \neq \emptyset$ and $\varrho: X \times X \rightarrow[1, \infty)$. Suppose that a function $d_{c}: X \times X \rightarrow[0, \infty)$ satisfies the following:

(CML1) $d_{c}(s, r)=0 \Rightarrow s=r$

(CML2) $d_{c}(s, r)=d_{c}(r, s)$;

(CML3) $d_{c}(s, r) \leq \varrho(s, z) d_{c}(s, z)+\varrho(z, r) d_{c}(z, r)$ for all $s, r, z \in X$.

Then $\left(X, d_{c}\right)$ is called a controlled metric-like space.

Example 1.4 ([25]) Let $X=\{0,1,2\}$. Define the function $d_{c}$ by

$$
d_{c}(0,0)=d_{c}(1,1)=0, \quad d_{c}(2,2)=\frac{1}{10}
$$

and

$$
d_{c}(0,1)=d_{c}(1,0)=1, \quad d_{c}(0,2)=d_{c}(2,0)=\frac{1}{2}, \quad d_{c}(1,2)=d_{c}(2,1)=\frac{2}{5} .
$$

Let $\varrho: X \times X \rightarrow[1, \infty)$ a symmetric function defined by

$$
\varrho(0,0)=\varrho(1,1)=\varrho(2,2)=\varrho(0,2)=1, \quad \varrho(1,2)=\frac{5}{4}, \quad \varrho(0,1)=\frac{11}{10} .
$$

Here $d_{c}$ is a controlled metric-like on $X$.

We have $d_{c}(2,2)=\frac{1}{10} \neq 0$, which implies that $\left(X, d_{c}\right)$ is not a controlled metric-type space.

Definition 1.5 ([25]) Let $\left(X, d_{c}\right)$ be a controlled metric-like space, and let $\left\{s_{n}\right\}_{n \geq 0}$ be a sequence in $X$.

(1) $\left\{s_{n}\right\}$ converges to $s$ in $X$ if and only if

$$
\lim _{n \rightarrow \infty} d_{c}\left(s_{n}, s\right)=d_{c}(s, s)
$$

Then we write $\lim _{n \rightarrow \infty} s_{n}=s$.

(2) $\left\{s_{n}\right\}$ is a Cauchy sequence if and only if $\lim _{n, m \rightarrow \infty} d_{c}\left(s_{n}, s_{m}\right)$ exists and is finite.

(3) We say that $\left(X, d_{c}\right)$ is complete if for every Cauchy sequence $\left\{s_{n}\right\}$, there is $s \in \chi$ such that

$$
\lim _{n \rightarrow \infty} d_{c}\left(s_{n}, s\right)=d_{c}(s, s)=\lim _{n, m \rightarrow \infty} d_{c}\left(s_{n}, s_{m}\right) .
$$

Definition 1.6 ([26]) Let $\left(X, d_{c}\right)$ be a controlled metric-like space. Let $s \in X$ and $\tau>0$.

(i) The open ball $B(s, \tau)$ is

$$
B(s, \tau)=\left\{y \in X,\left|d_{c}(s, r)-d_{c}(s, s)\right|<\tau\right\}
$$

We denote controlled metric-like spaces by $C M L S$.

Note that if $\mathfrak{T}$ is continuous at $\mathfrak{p}$ in the $\operatorname{CMLS}\left(X, d_{c}\right)$, then $\mathfrak{p}_{n} \rightarrow \mathfrak{p}$ implies that $\mathfrak{T p}_{n} \rightarrow \mathfrak{T p}$ as $n \rightarrow \infty$. 
Now let $\left(X, d_{c}\right)$ be a controlled metric-like space, and let $\mathfrak{T}: X \rightarrow X$. The following control functions were introduced by Sintunavarat et al. [27] (in this paper, we will exclude zero from their range):

$$
\mathrm{A}=\{\vartheta: X \rightarrow(0,1), \vartheta(\mathfrak{T} \mathfrak{p}) \leq \vartheta(\mathfrak{p}) \text { for all } \mathfrak{p} \in X\}
$$

and

$$
\mathrm{B}=\{\vartheta: X \rightarrow(0,1 / 2), \vartheta(\mathfrak{T} \mathfrak{p}) \leq \vartheta(\mathfrak{p}) \text { for all } \mathfrak{p} \in X\}
$$

\section{Main results}

Our first main result corresponds to a nonlinear Banach-type result on CMLS, which is also an extension of the results in [28].

Theorem 2.1 Let $\left(X, d_{c}\right)$ be a complete CMLS. Consider the mapping $\mathfrak{T}: X \rightarrow X$ such that

$$
d_{c}(\mathfrak{T} \mathfrak{p}, \mathfrak{T} \mathfrak{q}) \leq \vartheta(\mathfrak{p}) d_{c}(\mathfrak{p}, \mathfrak{q})
$$

for all $\mathfrak{p}, \mathfrak{q} \in X$, where $\vartheta \in A$. For $\mathfrak{p}_{0} \in X$, take $\mathfrak{p}_{n}=\mathfrak{T}^{n} \mathfrak{p}_{0}$. Suppose that

$$
\sup _{m \geq 1} \lim _{i \rightarrow \infty} \frac{\varrho\left(\mathfrak{p}_{i+1}, \mathfrak{p}_{i+2}\right)}{\varrho\left(\mathfrak{p}_{i}, \mathfrak{p}_{i+1}\right)} \varrho\left(\mathfrak{p}_{i+1}, \mathfrak{p}_{m}\right)<\frac{1}{\vartheta\left(\mathfrak{p}_{0}\right)} .
$$

Also, assume that for every $\mathfrak{p} \in X$, we have

$$
\lim _{n \rightarrow \infty} \varrho\left(\mathfrak{p}_{n}, \mathfrak{p}\right) \quad \text { and } \quad \lim _{n \rightarrow \infty} \varrho\left(\mathfrak{p}, \mathfrak{p}_{n}\right) \quad \text { exist and are finite. }
$$

Then $\mathfrak{T}$ has a unique fixed point.

Proof Consider the sequence $\left\{\mathfrak{p}_{n}=\mathfrak{T}^{n} \mathfrak{p}_{0}\right\}$. By $(2.1)$ we get

$$
d_{c}\left(\mathfrak{p}_{n}, \mathfrak{p}_{n+1}\right) \leq \vartheta\left(\mathfrak{p}_{n-1}\right) d_{c}\left(\mathfrak{p}_{n-1}, \mathfrak{p}_{n}\right) \quad \text { for all } n \geq 1
$$

Since $\vartheta \in \mathrm{A}$, we have

$$
d_{c}\left(\mathfrak{p}_{n}, \mathfrak{p}_{n+1}\right) \leq \vartheta\left(\mathfrak{p}_{0}\right) d_{c}\left(\mathfrak{p}_{n-1}, \mathfrak{p}_{n}\right) \quad \text { for all } n \geq 1
$$

By induction,

$$
d_{c}\left(\mathfrak{p}_{n}, \mathfrak{p}_{n+1}\right) \leq\left[\vartheta\left(\mathfrak{p}_{0}\right)\right]^{n} d_{c}\left(\mathfrak{p}_{0}, \mathfrak{p}_{1}\right) \quad \text { for all } n \geq 0
$$

Choose $k=: \vartheta\left(\mathfrak{p}_{0}\right) \in(0,1)$. For all natural numbers $n<m$, as in [24], we have

$$
\begin{aligned}
d_{c}\left(\mathfrak{p}_{n}, \mathfrak{p}_{m}\right) & \leq \varrho\left(\mathfrak{p}_{n}, \mathfrak{p}_{n+1}\right) d_{c}\left(\mathfrak{p}_{n}, \mathfrak{p}_{n+1}\right)+\varrho\left(\mathfrak{p}_{n+1}, \mathfrak{p}_{m}\right) d_{c}\left(\mathfrak{p}_{n+1}, \mathfrak{p}_{m}\right) \\
& \leq \varrho\left(\mathfrak{p}_{n}, \mathfrak{p}_{n+1}\right) k^{n} d_{c}\left(\mathfrak{p}_{0}, \mathfrak{p}_{1}\right)+\sum_{i=n+1}^{m-1}\left(\prod_{j=n+1}^{i} \varrho\left(\mathfrak{p}_{j}, \mathfrak{p}_{m}\right)\right) \varrho\left(\mathfrak{p}_{i}, \mathfrak{p}_{i+1}\right) k^{i} d_{c}\left(\mathfrak{p}_{0}, \mathfrak{p}_{1}\right) \\
& \leq \varrho\left(\mathfrak{p}_{n}, \mathfrak{p}_{n+1}\right) k^{n} d_{c}\left(\mathfrak{p}_{0}, \mathfrak{p}_{1}\right)+\sum_{i=n+1}^{m-1}\left(\prod_{j=0}^{i} \varrho\left(\mathfrak{p}_{j}, \mathfrak{p}_{m}\right)\right) \varrho\left(\mathfrak{p}_{i}, \mathfrak{p}_{i+1}\right) k^{i} d_{c}\left(\mathfrak{p}_{0}, \mathfrak{p}_{1}\right) .
\end{aligned}
$$


Let

$$
S_{p}=\sum_{i=0}^{p}\left(\prod_{j=0}^{i} \varrho\left(\mathfrak{p}_{j}, \mathfrak{p}_{m}\right)\right) \varrho\left(\mathfrak{p}_{i}, \mathfrak{p}_{i+1}\right) k^{i} .
$$

Hence we have

$$
d_{c}\left(\mathfrak{p}_{n}, \mathfrak{p}_{m}\right) \leq d_{c}\left(\mathfrak{p}_{0}, \mathfrak{p}_{1}\right)\left[k^{n} \varrho\left(\mathfrak{p}_{n}, \mathfrak{p}_{n+1}\right)+\left(S_{m-1}-S_{n}\right)\right] .
$$

Now by condition (2.2) and the ratio test, we deduce that $\lim _{n \rightarrow \infty} S_{n}$ exists, and therefore $\left\{S_{n}\right\}$ is a Cauchy sequence. Taking the limit in (2.5), we obtain

$$
\lim _{n, m \rightarrow \infty} d_{c}\left(\mathfrak{p}_{n}, \mathfrak{p}_{m}\right)=0
$$

Hence $\left\{\mathfrak{p}_{n}\right\}$ is a Cauchy sequence. Since $\left(X, d_{c}\right)$ is complete, we deduce that $\left\{\mathfrak{p}_{n}\right\}$ converges to some $u \in X$. We claim that $u$ is a fixed point of $\mathfrak{T}$. To prove this claim, we start by applying the triangle inequality of the $C M L S$ as follows:

$$
d_{c}\left(u, \mathfrak{p}_{n+1}\right) \leq \varrho\left(u, \mathfrak{p}_{n}\right) d_{c}\left(u, \mathfrak{p}_{n}\right)+\varrho\left(\mathfrak{p}_{n}, \mathfrak{p}_{n+1}\right) d_{c}\left(\mathfrak{p}_{n}, \mathfrak{p}_{n+1}\right)
$$

By (2.2), (2.3), and (2.6) we conclude that

$$
\lim _{n \rightarrow \infty} d_{c}\left(u, \mathfrak{p}_{n+1}\right)=0
$$

Thus

$$
\begin{aligned}
d_{c}(u, \mathfrak{T} u) & \leq \varrho\left(u, \mathfrak{p}_{n+1}\right) d_{c}\left(u, \mathfrak{p}_{n+1}\right)+\varrho\left(\mathfrak{p}_{n+1}, \mathfrak{T} u\right) d_{c}\left(\mathfrak{p}_{n+1}, \mathfrak{T} u\right) \\
& \leq \varrho\left(u, \mathfrak{p}_{n+1}\right) d_{c}\left(u, \mathfrak{p}_{n+1}\right)+\vartheta\left(\mathfrak{p}_{n}\right) \varrho\left(\mathfrak{p}_{n+1}, \mathfrak{T} u\right) d_{c}\left(\mathfrak{p}_{n}, u\right) \\
& \leq \varrho\left(u, \mathfrak{p}_{n+1}\right) d_{c}\left(u, \mathfrak{p}_{n+1}\right)+\vartheta\left(\mathfrak{p}_{0}\right) \varrho\left(\mathfrak{p}_{n+1}, \mathfrak{T} u\right) d_{c}\left(\mathfrak{p}_{n}, u\right) .
\end{aligned}
$$

Note that, as $n \rightarrow \infty$ in (2.3) and (2.7), we have $d_{c}(u, \mathfrak{T} u)=0$, that is, $\mathfrak{T} u=u$. Now we may assume that $\mathfrak{T}$ has fixed points $u$ and $v$. Hence

$$
d_{c}(u, v)=d_{c}(\mathfrak{T} u, \mathfrak{T} v) \leq \vartheta(u) d_{c}(u, v)<d_{c}(u, v)
$$

which leads us to a contradiction. Thereby $d_{c}(u, v)=0$, which implies $u=v$, as desired.

Next, we present the following example.

Example 2.2 Let $X=[0,1]$. Consider the CMLS $\left(X, d_{c}\right)$ defined by

$$
d_{c}(\mathfrak{p}, \mathfrak{q})=|\mathfrak{p}-\mathfrak{q}|^{2},
$$


where $\varrho(\mathfrak{p}, \mathfrak{q})=\mathfrak{p q}+1$ for $\mathfrak{p}, \mathfrak{q} \in X$. Take $\mathfrak{T} \mathfrak{p}=\frac{\mathfrak{p}^{2}}{4}$. Choose $\vartheta: X \rightarrow[0,1)$ as $\vartheta(\mathfrak{p})=\frac{\mathfrak{p}+1}{4}$. Then $\vartheta \in \mathrm{A}$. Take $\mathfrak{p}_{0}=0$, so (2.2) is satisfied. Let $\mathfrak{p}, \mathfrak{q} \in X$. Then

$$
\begin{aligned}
d_{c}(\mathfrak{T} \mathfrak{p}, \mathfrak{T} \mathfrak{q}) & =\frac{\left(\mathfrak{p}^{2}-\mathfrak{q}^{2}\right)^{2}}{16}=\frac{1}{16}|\mathfrak{p}-\mathfrak{q}|^{2}(\mathfrak{p}+\mathfrak{q})^{2} \\
& \leq \frac{1}{4}|\mathfrak{p}-\mathfrak{q}|^{2} \\
& \leq \frac{\mathfrak{p}+1}{4}|\mathfrak{p}-\mathfrak{q}|^{2} \\
& =\vartheta(\mathfrak{p}) d_{c}(\mathfrak{p}, \mathfrak{q}) .
\end{aligned}
$$

Note that all the hypotheses of Theorem 2.1 are satisfied. Thus there exists an element $u \in X$ such that $\mathfrak{T} u=u$, which is $u=0$.

In the following theorem, we propose a fixed point result using the nonlinear Kannantype contraction via the auxiliary function $\vartheta \in \mathrm{B}$.

Theorem 2.3 Let $\left(X, d_{c}\right)$ be a complete CMLS by the function $\varrho: X \times X \rightarrow[1, \infty)$. Let $\mathfrak{T}: X \rightarrow X$ where

$$
d_{c}(\mathfrak{T} \mathfrak{p}, \mathfrak{T} \mathfrak{q}) \leq \vartheta(\mathfrak{p})\left[d_{c}(\mathfrak{p}, \mathfrak{T} \mathfrak{p})+d_{c}(\mathfrak{q}, \mathfrak{T} \mathfrak{q})\right]
$$

for all $\mathfrak{p}, \mathfrak{q} \in X$, where $\vartheta \in \mathrm{B}$. For $\mathfrak{p}_{0} \in X$, take $\mathfrak{p}_{n}=\mathfrak{T}^{n} \mathfrak{p}_{0}$. Suppose that

$$
\sup _{m \geq 1} \lim _{i \rightarrow \infty} \frac{\varrho\left(\mathfrak{p}_{i+1}, \mathfrak{p}_{i+2}\right)}{\varrho\left(\mathfrak{p}_{i}, \mathfrak{p}_{i+1}\right)} \varrho\left(\mathfrak{p}_{i+1}, \mathfrak{p}_{m}\right)<\frac{1-\vartheta\left(\mathfrak{p}_{0}\right)}{\vartheta\left(\mathfrak{p}_{0}\right)}
$$

Also, assume that for every $\mathfrak{p} \in X$, we have

$$
\lim _{n \rightarrow \infty} \varrho\left(\mathfrak{p}, \mathfrak{p}_{n}\right) \quad \text { exists, is finite and } \quad \lim _{n \rightarrow \infty} \varrho\left(\mathfrak{p}_{n}, \mathfrak{p}\right)<\frac{1}{\vartheta\left(\mathfrak{p}_{0}\right)}
$$

Then there exists a unique fixed point of $\mathfrak{T}$.

Proof Let $\left\{\mathfrak{p}_{n}=\mathfrak{T p}_{n-1}\right\}$ be a sequence in $X$ satisfying hypotheses (2.9) and (2.10). From (2.8) we obtain

$$
\begin{aligned}
d_{c}\left(\mathfrak{p}_{n}, \mathfrak{p}_{n+1}\right) & =d_{c}\left(\mathfrak{T p}_{n-1}, \mathfrak{T} \mathfrak{p}_{n}\right) \\
& \leq \vartheta\left(\mathfrak{p}_{n-1}\right)\left[d_{c}\left(\mathfrak{p}_{n-1}, \mathfrak{T p}_{n-1}\right)+d_{c}\left(\mathfrak{p}_{n}, \mathfrak{T p}_{n}\right)\right] \\
& \leq \vartheta\left(\mathfrak{p}_{0}\right)\left[d_{c}\left(\mathfrak{p}_{n-1}, \mathfrak{p}_{n}\right)+d_{c}\left(\mathfrak{p}_{n}, \mathfrak{p}_{n+1}\right)\right] .
\end{aligned}
$$

Consider $a=\vartheta\left(\mathfrak{p}_{0}\right)$. Then $d_{c}\left(\mathfrak{p}_{n}, \mathfrak{p}_{n+1}\right) \leq \frac{a}{1-a} d_{c}\left(\mathfrak{p}_{n-1}, \mathfrak{p}_{n}\right)$. By induction we get

$$
d_{c}\left(\mathfrak{p}_{n}, \mathfrak{p}_{n+1}\right) \leq\left(\frac{a}{1-a}\right)^{n} d_{c}\left(\mathfrak{p}_{1}, \mathfrak{p}_{0}\right), \quad \forall n \geq 0
$$

For all natural numbers $n, m$, we have

$$
d_{c}\left(\mathfrak{p}_{n}, \mathfrak{p}_{m}\right) \leq \varrho\left(\mathfrak{p}_{n}, \mathfrak{p}_{n+1}\right) d_{c}\left(\mathfrak{p}_{n}, \mathfrak{p}_{n+1}\right)+\varrho\left(\mathfrak{p}_{n+1}, \mathfrak{p}_{m}\right) d_{c}\left(\mathfrak{p}_{n+1}, \mathfrak{p}_{m}\right)
$$


Following the steps of the proof of Theorem 2.1, we deduce

$$
\begin{aligned}
d_{c}\left(\mathfrak{p}_{n}, \mathfrak{p}_{m}\right) \leq & \varrho\left(\mathfrak{p}_{n}, \mathfrak{p}_{n+1}\right) d_{c}\left(\mathfrak{p}_{n}, \mathfrak{p}_{n+1}\right)+\sum_{i=n+1}^{m-2}\left(\prod_{j=n+1}^{i} \varrho\left(\mathfrak{p}_{j}, \mathfrak{p}_{m}\right)\right) \varrho\left(\mathfrak{p}_{i}, \mathfrak{p}_{i+1}\right) d_{c}\left(\mathfrak{p}_{i}, \mathfrak{p}_{i+1}\right) \\
& +\prod_{k=n+1}^{m-1} \varrho\left(\mathfrak{p}_{k}, \mathfrak{p}_{m}\right) d_{c}\left(\mathfrak{p}_{m-1}, \mathfrak{p}_{m}\right) \\
\leq & \varrho\left(\mathfrak{p}_{n}, \mathfrak{p}_{n+1}\right)\left(\frac{a}{1-a}\right)^{n} d_{c}\left(\mathfrak{p}_{0}, \mathfrak{p}_{1}\right) \\
& +\sum_{i=n+1}^{m-2}\left(\prod_{j=n+1}^{i} \varrho\left(\mathfrak{p}_{j}, \mathfrak{p}_{m}\right)\right) \varrho\left(\mathfrak{p}_{i}, \mathfrak{p}_{i+1}\right)\left(\frac{a}{1-a}\right)^{i} d_{c}\left(\mathfrak{p}_{0}, \mathfrak{p}_{1}\right) \\
& +\prod_{i=n+1}^{m-1} \varrho\left(\mathfrak{p}_{i}, \mathfrak{p}_{m}\right)\left(\frac{a}{1-a}\right)^{m-1} d_{c}\left(\mathfrak{p}_{0}, \mathfrak{p}_{1}\right) .
\end{aligned}
$$

Since $0 \leq a<\frac{1}{2}$, we have $\frac{a}{1-a} \in(0,1)$. Therefore $\left\{\mathfrak{p}_{n}\right\}$ is a Cauchy sequence, and since $\left(X, d_{c}\right)$ is a complete $C M L S,\left\{\mathfrak{p}_{n}\right\}$ converges to some $u \in X$. Suppose that $\mathfrak{T} u \neq u$. Then

$$
\begin{aligned}
0 & <d_{c}(u, \mathfrak{T} u) \leq \varrho\left(u, \mathfrak{p}_{n+1}\right) d_{c}\left(u, \mathfrak{p}_{n+1}\right)+\varrho\left(\mathfrak{p}_{n+1}, \mathfrak{T} u\right) d_{c}\left(\mathfrak{p}_{n+1}, \mathfrak{T} u\right) \\
& \leq \varrho\left(u, \mathfrak{p}_{n+1}\right) d_{c}\left(u, \mathfrak{p}_{n+1}\right)+\varrho\left(\mathfrak{p}_{n+1}, \mathfrak{T} u\right) \vartheta\left(\mathfrak{p}_{n}\right)\left[d_{c}\left(\mathfrak{p}_{n}, \mathfrak{p}_{n+1}\right)+d_{c}(u, \mathfrak{T} u)\right] \\
& \leq \varrho\left(u, \mathfrak{p}_{n+1}\right) d_{c}\left(u, \mathfrak{p}_{n+1}\right)+\varrho\left(\mathfrak{p}_{n+1}, \mathfrak{T} u\right) \vartheta\left(\mathfrak{p}_{0}\right)\left[d_{c}\left(\mathfrak{p}_{n}, \mathfrak{p}_{n+1}\right)+d_{c}(u, \mathfrak{T} u)\right]
\end{aligned}
$$

As $n \rightarrow \infty$ in (2.12) and by (2.10), we conclude that $0<d_{c}(u, \mathfrak{T} u)<d_{c}(u, \mathfrak{T} u)$, which leads us to a contradiction. Thereby $\mathfrak{T} u=u$. Now we may assume that $\mathfrak{T}$ has fixed points $u$ and $v$. Thus

$$
\begin{aligned}
d_{c}(u, v) & =d_{c}(\mathfrak{T} u, \mathfrak{T} v) \leq \vartheta(u)\left[d_{c}(u, \mathfrak{T} u)+d_{c}(v, \mathfrak{T} v)\right] \\
& =\vartheta(u)\left[d_{c}(u, u)+d_{c}(v, v)\right]=0 .
\end{aligned}
$$

Hence $u=v$. Therefore the fixed point is unique, as required.

Example 2.4 Consider $X=\{0,1,2\}$. Take the controlled metric-like $d_{c}$ defined as

$$
d_{c}(0,1)=\frac{1}{2}, \quad d_{c}(0,2)=\frac{11}{20}, \quad d_{c}(1,2)=\frac{3}{20}
$$

Let $\varrho: X \times X \rightarrow[1, \infty)$ be defined by

$$
\begin{aligned}
& \varrho(0,0)=\varrho(1,1)=\varrho(2,2)=\varrho(1,2)=\varrho(2,1)=1, \\
& \varrho(0,2)=\varrho(2,0)=2, \quad \varrho(0,1)=\varrho(1,0)=\frac{3}{2} .
\end{aligned}
$$

Let $\mathfrak{T}: X \rightarrow X$ be given by 
Let $\vartheta: X \rightarrow\left[0, \frac{1}{2}\right)$ be given by $\vartheta(0)=\frac{99}{200}, \vartheta(1)=\frac{3}{10}$, and $\vartheta(2)=\frac{49}{100}$. Then $\vartheta \in \mathrm{B}$. Take $\mathfrak{p}_{0}=0$, so that $(2.9)$ is satisfied.

Also, it is easy to see that (2.8) holds. By Theorem 2.3 there exists a unique $u$ such that $\mathfrak{T} u=u$, that is, $u=1$.

Now,we again give a response to an open question in [24], which is a study of a nonlinear Chatterjea-type contraction via an auxiliary function $\vartheta \in \mathrm{B}$.

Theorem 2.5 Let $\left(X, d_{c}\right)$ be a complete CMLS by the function

$$
\begin{aligned}
& \varrho: X \times X \rightarrow[1, \infty) \\
& \text { Let } \mathfrak{T}: X \rightarrow X \text { be such that } d_{c}(\mathfrak{T} \mathfrak{p}, \mathfrak{T} \mathfrak{q}) \leq \vartheta(\mathfrak{p})\left[d_{c}(\mathfrak{p}, \mathfrak{T} \mathfrak{q})+d_{c}(\mathfrak{q}, \mathfrak{T} \mathfrak{p})\right]
\end{aligned}
$$

for all $\mathfrak{p}, \mathfrak{q} \in X$, where $\vartheta \in \mathrm{B}$. For $\mathfrak{p}_{0} \in X$, take $\mathfrak{p}_{n}=\mathfrak{T}^{n} \mathfrak{p}_{0}$. Suppose that

$$
\begin{aligned}
& \sup _{i \geq 1} \varrho\left(\mathfrak{p}_{i-1}, \mathfrak{p}_{i}\right)=\beta \quad \text { (exists and is finite), } \\
& 0<\vartheta\left(\mathfrak{p}_{0}\right)<\frac{1}{2 \beta}
\end{aligned}
$$

and

$$
\sup _{m \geq 1} \lim _{i \rightarrow \infty} \frac{\varrho\left(\mathfrak{p}_{i+1}, \mathfrak{p}_{i+2}\right)}{\varrho\left(\mathfrak{p}_{i}, \mathfrak{p}_{i+1}\right)} \varrho\left(\mathfrak{p}_{i+1}, \mathfrak{p}_{m}\right)<\frac{\beta \vartheta\left(\mathfrak{p}_{0}\right)}{1-\beta \vartheta\left(\mathfrak{p}_{0}\right)}
$$

Also, assume that $d_{c}$ is continuous with respect to the first variable and that for every $\mathfrak{p} \in X$,

$$
\lim _{n \rightarrow \infty} \varrho\left(\mathfrak{p}, \mathfrak{p}_{n}\right) \quad \text { exists, is finite, and } \lim _{n \rightarrow \infty} \varrho\left(\mathfrak{p}_{n}, \mathfrak{p}\right)<\frac{1}{\vartheta\left(\mathfrak{p}_{0}\right)}
$$

Then $\boldsymbol{T}$ possesses a unique fixed point in $X$.

Proof Consider the sequence $\left\{\mathfrak{p}_{n}=\mathfrak{T p}_{n-1}\right\}$ in $X$ satisfying hypotheses (2.14), (2.15), (2.16), and (2.17). From (2.13) and (2.14) we obtain

$$
\begin{aligned}
d_{c}\left(\mathfrak{p}_{n}, \mathfrak{p}_{n+1}\right) & =d_{c}\left(\mathfrak{T p}_{n-1}, \mathfrak{T}_{\mathfrak{p}_{n}}\right) \\
& \leq \vartheta\left(\mathfrak{p}_{n-1}\right)\left[d_{c}\left(\mathfrak{p}_{n-1}, \mathfrak{T p}_{n}\right)+d_{c}\left(\mathfrak{p}_{n}, \mathfrak{T p}_{n-1}\right)\right] \\
& =\vartheta\left(\mathfrak{p}_{n-1}\right) d_{c}\left(\mathfrak{p}_{n-1}, \mathfrak{p}_{n+1}\right) \\
& \leq \vartheta\left(\mathfrak{p}_{0}\right)\left[\varrho\left(\mathfrak{p}_{n-1}, \mathfrak{p}_{n}\right) d_{c}\left(\mathfrak{p}_{n-1}, \mathfrak{p}_{n}\right)+\varrho\left(\mathfrak{p}_{n}, \mathfrak{p}_{n+1}\right) d_{c}\left(\mathfrak{p}_{n}, \mathfrak{p}_{n+1}\right)\right] \\
& \leq \beta \vartheta\left(\mathfrak{p}_{0}\right)\left[d_{c}\left(\mathfrak{p}_{n-1}, \mathfrak{p}_{n}\right)+d_{c}\left(\mathfrak{p}_{n}, \mathfrak{p}_{n+1}\right)\right] .
\end{aligned}
$$

Let $b=\frac{\beta \vartheta\left(\mathfrak{p}_{0}\right)}{1-\beta \vartheta\left(\mathfrak{p}_{0}\right)}$. By $(2.15)$ we have $b \in(0,1)$. Then $d_{c}\left(\mathfrak{p}_{n}, \mathfrak{p}_{n+1}\right) \leq b d_{c}\left(\mathfrak{p}_{n-1}, \mathfrak{p}_{n}\right)$. By induction we get

$$
d_{c}\left(\mathfrak{p}_{n}, \mathfrak{p}_{n+1}\right) \leq b^{n} d_{c}\left(\mathfrak{p}_{0}, \mathfrak{p}_{1}\right), \quad \forall n \geq 0
$$


For all natural numbers $n, m$, we have

$$
d_{c}\left(\mathfrak{p}_{n}, \mathfrak{p}_{m}\right) \leq \varrho\left(\mathfrak{p}_{n}, \mathfrak{p}_{n+1}\right) d_{c}\left(\mathfrak{p}_{n}, \mathfrak{p}_{n+1}\right)+\varrho\left(\mathfrak{p}_{n+1}, \mathfrak{p}_{m}\right) d_{c}\left(\mathfrak{p}_{n+1}, \mathfrak{p}_{m}\right)
$$

Following the steps of the proof of Theorem 2.1, we get

$$
\begin{aligned}
d_{c}\left(\mathfrak{p}_{n}, \mathfrak{p}_{m}\right) \leq & \varrho\left(\mathfrak{p}_{n}, \mathfrak{p}_{n+1}\right) d_{c}\left(\mathfrak{p}_{n}, \mathfrak{p}_{n+1}\right)+\sum_{i=n+1}^{m-2}\left(\prod_{j=n+1}^{i} \varrho\left(\mathfrak{p}_{j}, \mathfrak{p}_{m}\right)\right) \varrho\left(\mathfrak{p}_{i}, \mathfrak{p}_{i+1}\right) d_{c}\left(\mathfrak{p}_{i}, \mathfrak{p}_{i+1}\right) \\
& +\prod_{k=n+1}^{m-1} \varrho\left(\mathfrak{p}_{k}, \mathfrak{p}_{m}\right) d_{c}\left(\mathfrak{p}_{m-1}, \mathfrak{p}_{m}\right) \\
\leq & \varrho\left(\mathfrak{p}_{n}, \mathfrak{p}_{n+1}\right)\left(b^{n} d_{c}\left(\mathfrak{p}_{0}, \mathfrak{p}_{1}\right)+\sum_{i=n+1}^{m-2}\left(\prod_{j=n+1}^{i} \varrho\left(\mathfrak{p}_{j}, \mathfrak{p}_{m}\right)\right) \varrho\left(\mathfrak{p}_{i}, \mathfrak{p}_{i+1}\right) b^{i} d_{c}\left(\mathfrak{p}_{0}, \mathfrak{p}_{1}\right)\right. \\
& +\prod_{i=n+1}^{m-1} \varrho\left(\mathfrak{p}_{i}, \mathfrak{p}_{m}\right) b^{m-1} d_{c}\left(\mathfrak{p}_{0}, \mathfrak{p}_{1}\right) .
\end{aligned}
$$

This implies that $\left\{\mathfrak{p}_{n}\right\}$ is a Cauchy sequence $C M L S\left(X, d_{c}\right)$. Since the space is complete, the sequence $\left\{\mathfrak{p}_{n}\right\}$ converges to some $u \in X$. Now suppose that $\mathfrak{T} u \neq u$. Then

$$
\begin{aligned}
0 & <d_{c}(u, \mathfrak{T} u) \leq \varrho\left(u, \mathfrak{p}_{n+1}\right) d_{c}\left(u, \mathfrak{p}_{n+1}\right)+\varrho\left(\mathfrak{p}_{n+1}, \mathfrak{T} u\right) d_{c}\left(\mathfrak{p}_{n+1}, \mathfrak{T} u\right) \\
& \leq \varrho\left(u, \mathfrak{p}_{n+1}\right) d_{c}\left(u, \mathfrak{p}_{n+1}\right)+\varrho\left(\mathfrak{p}_{n+1}, \mathfrak{T} u\right) \vartheta\left(\mathfrak{p}_{n}\right)\left[d_{c}\left(\mathfrak{p}_{n}, \mathfrak{T} u\right)+d_{c}\left(u, \mathfrak{p}_{n+1}\right)\right] \\
& \leq \varrho\left(u, \mathfrak{p}_{n+1}\right) d_{c}\left(u, \mathfrak{p}_{n+1}\right)+\varrho\left(\mathfrak{p}_{n+1}, \mathfrak{T} u\right) \vartheta\left(\mathfrak{p}_{0}\right)\left[d_{c}\left(\mathfrak{p}_{n}, \mathfrak{T} u\right)+d_{c}\left(u, \mathfrak{p}_{n+1}\right)\right]
\end{aligned}
$$

As $n \rightarrow \infty$ in (2.19), by (2.17) and using the continuity of $d_{c}$ with respect to its first variable, we deduce that $0<d_{c}(u, \mathfrak{T} u)<d_{c}(u, \mathfrak{T} u)$, which leads us to a contradiction. Thus $\mathfrak{T} u=u$.

Now let us assume that $\mathfrak{T}$ has fixed points $u$ and $v$. Then

$$
\begin{aligned}
d_{c}(u, v) & =d_{c}(\mathfrak{T} u, \mathfrak{T} v) \leq \vartheta(u)\left[d_{c}(u, \mathfrak{T} v)+d_{c}(v, \mathfrak{T} u)\right] \\
& =\vartheta(u)\left[d_{c}(u, u)+d_{c}(v, v)\right]=0 .
\end{aligned}
$$

Therefore $u=v$, and thus the fixed point of $\mathfrak{T}$ is unique.

Now we introduce cyclical orbital contractions in the class of CMLS.

Definition 2.6 Let $U$ and $V$ be two nonempty subsets of a $C M L S\left(X, d_{c}\right)$. Let $\mathfrak{T}: U \cup V \rightarrow$ $U \cup V$ be a cyclic mapping (i.e., $\mathfrak{T}(U) \subseteq V$ and $\mathfrak{T} V \subseteq U$ ) such that for some $\mathfrak{p} \in U$, there exists $k_{\mathfrak{p}} \in(0,1)$ such that

$$
d_{c}\left(\mathfrak{T}^{2 n} \mathfrak{p}, \mathfrak{T} \mathfrak{q}\right) \leq k_{\mathfrak{p}} d_{c}\left(\mathfrak{T}^{2 n-1} \mathfrak{p}, \mathfrak{q}\right)
$$

where $n=1,2, \ldots$ and $\mathfrak{q} \in U$. Then $\mathfrak{T}$ is called a controlled cyclic orbital contraction mapping.

Finally, we prove the following result. 
Theorem 2.7 Let $U$ and $V$ be two nonempty closed subsets of a complete CMLS $\left(X, d_{c}\right)$. Let $\mathfrak{T}: X \rightarrow X$ be a controlled cyclic orbital contraction mapping. For $\mathfrak{p}_{0} \in U$, take $\mathfrak{p}_{n}=\mathfrak{T}^{n} \mathfrak{p}_{0}$. Suppose that

$$
\sup _{m \geq 1} \lim _{i \rightarrow \infty} \frac{\varrho\left(\mathfrak{p}_{i+1}, \mathfrak{p}_{i+2}\right)}{\varrho\left(\mathfrak{p}_{i}, \mathfrak{p}_{i+1}\right)} \varrho\left(\mathfrak{p}_{i+1}, \mathfrak{p}_{m}\right)<\frac{1}{k_{\mathfrak{p}_{0}}} .
$$

Also, assume that for every $\mathfrak{p} \in X$,

$$
\lim _{n \rightarrow \infty} \varrho\left(\mathfrak{p}_{n}, \mathfrak{p}\right) \quad \text { and } \quad \lim _{n \rightarrow \infty} \varrho\left(\mathfrak{p}, \mathfrak{p}_{n}\right) \quad \text { exist and are finite. }
$$

Then $U \cap V$ is nonempty, and $\mathfrak{T}$ has a unique fixed point.

Proof Suppose there exists $\mathfrak{p}$ (say $\mathfrak{p}_{0}$ ) in $U$ satisfying (2.20). Define the iterative sequence $\left\{\mathfrak{p}_{n}=\mathfrak{T}^{n} \mathfrak{p}_{0}\right\}$. Since $\mathfrak{p}_{0} \in U$ and $\mathfrak{T}$ is cyclic, we have

$$
\mathfrak{p}_{2 n} \in U \quad \text { and } \quad \mathfrak{p}_{2 n+1} \in V \quad \text { for all } n \geq 0 .
$$

By (2.20) we get

$$
d_{c}\left(\mathfrak{T}^{2} \mathfrak{p}, \mathfrak{T} \mathfrak{p}\right) \leq k_{\mathfrak{p}} d_{c}(\mathfrak{T} \mathfrak{p}, \mathfrak{p})
$$

Again,

$$
d_{c}\left(\mathfrak{T}^{3} \mathfrak{p}, \mathfrak{T}^{2} \mathfrak{p}\right)=d_{c}\left(\mathfrak{T}^{2} \mathfrak{p}, \mathfrak{T}\left(\mathfrak{T}^{2} \mathfrak{p}\right)\right) \leq k_{\mathfrak{p}} d_{c}\left(\mathfrak{T} \mathfrak{p}, \mathfrak{T}^{2} \mathfrak{p}\right) \leq\left(k_{\mathfrak{p}}\right)^{2} d_{c}(\mathfrak{T} \mathfrak{p}, \mathfrak{p}) .
$$

By induction we obtain that

$$
d_{c}\left(\mathfrak{p}_{n}, \mathfrak{p}_{n+1}\right) \leq\left[k_{\mathfrak{p}}\right]^{n} d_{c}\left(\mathfrak{p}_{0}, \mathfrak{p}_{1}\right) \quad \text { for all } n \geq 0
$$

Similarly to the proof of Theorem 2.1, we can easily deduce that

$$
\lim _{n, m \rightarrow \infty} d_{c}\left(\mathfrak{p}_{n}, \mathfrak{p}_{m}\right)=0
$$

that is, $\left\{\mathfrak{p}_{n}\right\}$ is a Cauchy sequence in the complete $\operatorname{CMLS}\left(X, d_{c}\right)$, so $\left\{\mathfrak{p}_{n}\right\}$ converges to some $u \in X$. Since $\left\{\mathfrak{T}^{2 n} \mathfrak{p}\right\}$ is in $U$ and $U$ is closed, the limit $u$ is in $S_{1}$. Similarly, $\left\{\mathfrak{T}^{2 n-1} \mathfrak{p}\right\}$ is in the closed subset $V$, so $u \in V$, that is, $u \in U \cap V$, and hence $U \cap V$ is not empty. Let us prove that $u$ is a fixed point of $\mathfrak{T}$. We have

$$
d_{c}\left(u, \mathfrak{p}_{n+1}\right) \leq \varrho\left(u, \mathfrak{p}_{n}\right) d_{c}\left(u, \mathfrak{p}_{n}\right)+\varrho\left(\mathfrak{p}_{n}, \mathfrak{p}_{n+1}\right) d_{c}\left(\mathfrak{p}_{n}, \mathfrak{p}_{n+1}\right)
$$

Using (2.21), (2.22), and (2.25), we get that

$$
\lim _{n \rightarrow \infty} d_{c}\left(u, \mathfrak{p}_{n+1}\right)=0
$$


By (2.20) we deduce

$$
\begin{aligned}
d_{c}(u, \mathfrak{T} u) & \leq \varrho\left(u, \mathfrak{T}^{2 n} \mathfrak{p}\right) d_{c}\left(u, \mathfrak{T}^{2 n} \mathfrak{p}\right)+\varrho\left(\mathfrak{T}^{2 n} \mathfrak{p}, \mathfrak{T} u\right) d_{c}\left(\mathfrak{T}^{2 n} \mathfrak{p}, \mathfrak{T} u\right) \\
& \leq \varrho\left(u, \mathfrak{T}^{2 n} \mathfrak{p}\right) d_{c}\left(u, \mathfrak{T}^{2 n} \mathfrak{p}\right)+k_{\mathfrak{p}} \varrho\left(\mathfrak{T}^{2 n} \mathfrak{p}, \mathfrak{T} u\right) d_{c}\left(\mathfrak{T}^{2 n-1} \mathfrak{p}, u\right) \\
& =\varrho\left(u, \mathfrak{p}_{n+1}\right) d_{c}\left(u, \mathfrak{p}_{n+1}\right)+k_{\mathfrak{p}} \varrho\left(\mathfrak{p}_{n+1}, \mathfrak{T} u\right) d_{c}\left(\mathfrak{p}_{2 n-1}, u\right) .
\end{aligned}
$$

Taking the limit as $n \rightarrow \infty$ and using (2.22) and (2.26), we deduce that $d_{c}(u, \mathfrak{T} u)=0$, that is, $\mathfrak{T} u=u$. Finally, assume that $\mathfrak{T}$ has two fixed points, say $u$ and $v$ (they are in $U$ ). Then

$$
d_{c}(u, v)=d_{c}(\mathfrak{T} u, \mathfrak{T} v)=d_{c}\left(\mathfrak{T}^{2 n} u, \mathfrak{T} v\right) \leq k_{u} d_{c}\left(\mathfrak{T}^{2 n-1} u, v\right)=k_{u} d_{c}(u, v),
$$

which holds unless $d_{c}(u, v)=0$, so $u=v$. Hence $\mathfrak{T}$ has a unique fixed point.

The following example illustrates Theorem 2.7.

Example 2.8 Let $X=U \cup V$, where $U=\left[\frac{1}{4}, \frac{1}{2}\right]$ and $V=\left[\frac{1}{2}, 1\right]$. Consider the controlled metric-like $d_{c}$ defined as

$$
d_{c}(\mathfrak{p}, \mathfrak{q})=|\mathfrak{p}-\mathfrak{q}|^{2},
$$

where $\varrho(\mathfrak{p}, \mathfrak{q})=\mathfrak{p q}+1$ for $\mathfrak{p}, \mathfrak{q} \in X$. Take $\mathfrak{T} \mathfrak{p}=\frac{1}{2}$ if $\mathfrak{p} \in U$ and $\mathfrak{T} \mathfrak{p}=\frac{\mathfrak{p}}{2}$ if $\mathfrak{p} \in V \backslash\left\{\frac{1}{2}\right\}$. Now let $k_{\mathfrak{p}}: X \rightarrow[0,1]$ be defined as $k_{\mathfrak{p}}=\frac{\mathfrak{p}+1}{2}$. Note that for all $\mathfrak{p} \in U$, we have

$$
\mathfrak{T} \mathfrak{p}=\frac{1}{2}, \quad \mathfrak{T}^{2} \mathfrak{p}=\frac{1}{2}, \quad \ldots, \quad \mathfrak{T}^{2 n-1} \mathfrak{p}=\frac{1}{2}, \quad \mathfrak{T}^{2 n} \mathfrak{p}=\frac{1}{2}, \quad \ldots
$$

For all $\mathfrak{q} \in U$, using the fact that

$$
d_{c}\left(\mathfrak{T}^{2 n} \mathfrak{p}, \mathfrak{T} \mathfrak{q}\right)=d_{c}\left(\frac{1}{2}, \frac{1}{2}\right)=0,
$$

we deduce that

$$
d_{c}\left(\mathfrak{T}^{2 n} \mathfrak{p}, \mathfrak{T} \mathfrak{q}\right) \leq k_{\mathfrak{p}} d_{c}\left(\mathfrak{T}^{2 n-1} \mathfrak{p}, \mathfrak{q}\right)
$$

It is not difficult to see that $\mathfrak{T}$ satisfies all the hypotheses of Theorem 2.7. Therefore $\mathfrak{T}$ has a unique fixed point $u=\frac{1}{2}$.

\section{Fredholm-type integral equation}

Consider the set $X=C([0,1],(-\infty, \infty))$ and the following Fredholm-type integral equation:

$$
\mathfrak{p}^{\prime}(t)=\int_{0}^{1} \mathbb{S}\left(t, s, \mathfrak{p}^{\prime}(t)\right) d s \quad \text { for } t \in[0,1]
$$

where $\mathbb{S}\left(t, s, \mathfrak{p}^{\prime}(t)\right)$ is a continuous function from $[0,1]^{2}$ into $\mathbb{R}$. Now define

$$
\begin{aligned}
d_{c}: X \times X & \longrightarrow \mathbb{R}^{+} \\
(\mathfrak{p}, \mathfrak{q}) & \mapsto \sup _{t \in[0,1]}\left(\frac{\left|\mathfrak{p}^{\prime}(t)\right|+|\mathfrak{q}(t)|}{2}\right) .
\end{aligned}
$$


Note that $\left(X, d_{c}\right)$ is a complete $C M L S$, where

$$
\varrho(\mathfrak{p}, \mathfrak{q})=2
$$

Theorem 3.1 Assume that for all $\mathfrak{p}, \mathfrak{q} \in X$,

(1) $\left|\mathbb{S}\left(t, s, \mathfrak{p}^{\prime}(t)\right)\right|+|\mathbb{S}(t, s, \mathfrak{q}(t))| \leq \vartheta\left(\sup _{t \in[0,1]}\left(\left|\mathfrak{p}^{\prime}(t)\right|+|\mathfrak{q}(t)|\right)\right)\left(\left|\mathfrak{p}^{\prime}(t)\right|+|\mathfrak{q}(t)|\right)$ for some $\vartheta \in \mathrm{B}$.

(2) $\mathbb{S}\left(t, s, \int_{0}^{1} \mathbb{S}\left(t, s, \mathfrak{p}^{\prime}(t)\right) d s\right)<\mathbb{S}\left(t, s, \mathfrak{p}^{\prime}(t)\right)$ for all $t, s$.

Then the integral equation (3.1) has a unique solution.

Proof Let $\mho: X \longrightarrow X$ be defined by $\mho \mathfrak{p}^{\prime}(t)=\int_{0}^{1} \mathbb{S}\left(t, s, \mathfrak{p}^{\prime}(t)\right) d s$. Then

$$
d_{c}\left(\mho \mathfrak{p}^{\prime}, \mho \mathfrak{q}\right)=\sup _{t \in[0,1]}\left(\frac{\left|\mho \mho \mathfrak{p}^{\prime}(t)\right|+|\mho \mathfrak{q}(t)|}{2}\right)
$$

Now we have

$$
\begin{aligned}
d_{c}\left(\mho \mathfrak{p}^{\prime}(t), \mho \mathfrak{q}(t)\right) & =\frac{\left|\mho \mathfrak{p}^{\prime}(t)\right|+|\mho \mathfrak{q}(t)|}{2} \\
& =\frac{\left|\int_{0}^{1} \mathbb{S}\left(t, s, \mathfrak{p}^{\prime}(t)\right) d s\right|+\left|\int_{0}^{1} \mathbb{S}(t, s, \mathfrak{q}(t)) d s\right|}{2} \\
& \leq \frac{\int_{0}^{1}\left|\mathbb{S}\left(t, s, \mathfrak{p}^{\prime}(t)\right)\right| d s+\int_{0}^{1}|\mathbb{S}(t, s, \mathfrak{q}(t))| d s}{2} \\
& =\frac{\int_{0}^{1}\left(\left|\mathbb{S}\left(t, s, \mathfrak{p}^{\prime}(t)\right)\right|+|\mathbb{S}(t, s, \mathfrak{q}(t))|\right) d s}{2} \\
& \leq \frac{\int_{0}^{1} \vartheta\left(\sup _{t \in[0,1]}\left(\left|\mathfrak{p}^{\prime}(t)\right|+|\mathfrak{q}(t)|\right)\right)\left(\left|\mathfrak{p}^{\prime}(t)\right|+|\mathfrak{q}(t)|\right) d s}{2} \\
& \leq \vartheta\left(\sup _{t \in[0,1]}\left(\left|\mathfrak{p}^{\prime}(t)\right|+|\mathfrak{q}(t)|\right)\right) d_{c}\left(\mathfrak{p}^{\prime}(t), \mathfrak{q}(t)\right) .
\end{aligned}
$$

Thus $d_{c}\left(\mho \mathfrak{p}^{\prime}, \mho \mathfrak{q}\right) \leq \vartheta\left(\sup _{t \in[0,1]}\left(\left|\mathfrak{p}^{\prime}(t)\right|+|\mathfrak{q}(t)|\right)\right) d_{c}\left(\mathfrak{p}^{\prime}, \mathfrak{q}\right)$. Also, notice that

$$
\varrho(\mathfrak{p}, \mathfrak{q})<\frac{1}{\vartheta\left(\sup _{t \in[0,1]}\left(\left|\mathfrak{p}^{\prime}(t)\right|+|\mathfrak{q}(t)|\right)\right)}
$$

Therefore all the hypotheses of Theorem 2.1 are satisfied, and hence equation (3.1) has a unique solution.

\section{Conclusion}

We have proved the existence and uniqueness of a fixed point for a self-mapping in controlled metric-like spaces under different nonlinear contractions with a control function. Also, we present an application of our results to Fredholm-type integral equations. Moreover, we would like to bring the reader's attention to the following question.

Question 4.1 Under what conditions we can obtain the same results for a self-mapping in double controlled metric-like spaces [26]? 


\section{Acknowledgements}

The first and the second authors would like to thank Prince Sultan University for funding this work through research group Nonlinear Analysis Methods in Applied Mathematics (NAMAM) group number RG-DES-2017-01-17.

\section{Funding}

Not applicable.

\section{Availability of data and materials}

Not applicable.

\section{Competing interests}

The authors declare that they have no competing interests.

\section{Authors' contributions}

All authors read and approved the final manuscript.

\section{Author details}

'Department of Mathematics and General Sciences, Prince Sultan University, Riyadh, 11586, Saudi Arabia. ${ }^{2}$ Department of Medical Research, China Medical University, Taichung 40402, Taiwan. ${ }^{3}$ Department of Mathematics, Faculty of Science, College of Science and Arts, Al Qassim University, Al-Asyah, Saudi Arabia. ${ }^{4}$ Department of Mathematics, Saint Paul College, Saint Paul, Minnesota 55102, United States.

\section{Publisher's Note}

Springer Nature remains neutral with regard to jurisdictional claims in published maps and institutional affiliations.

Received: 2 March 2021 Accepted: 8 July 2021 Published online: 31 July 2021

\section{References}

1. Marasi, H.R., Aydi, H.: Existence and uniqueness results for two-term nonlinear fractional differential equations via a fixed point technique. J. Math. 2021, Article ID 6670176 (2021)

2. Aydi, H., Bota, M.F., Karapınar, E., Moradi, S.: A common fixed point for weak $\phi$-contractions on $b$-metric spaces. Fixed Point Theory 13(2), 337-346 (2012)

3. Afshari, H., Aydi, H., Karapınar, E.: On generalized $\alpha-\psi$-Geraghty contractions on $b$-metric spaces. Georgian Math. J. 27(1), 9-21 (2021)

4. Karapınar, E., Chifu, C.: Results in wt-distance over b-metric spaces. Mathematics 8(2), 220 (2020)

5. Ameer, E., Aydi, H., Arshad, M., Alsamir, H., Noorani, M.S.: Hybrid multivalued type contraction mappings in $\alpha_{K}$-complete partial $b$-metric spaces and applications. Symmetry 11(1), 86 (2019)

6. Aydi, H., Karapınar, E., Bota, M.F., Mitrović, S.: A fixed point theorem for set-valued quasi-contractions in $b$-metric spaces. Fixed Point Theory Appl. 2012, 88 (2012)

7. Patle, P., Patel, D., Aydi, H., Radenović, S.: On $\mathrm{H}^{+}$-type multivalued contractions and applications in symmetric and probabilistic spaces. Mathematics 7(2), 144 (2019)

8. Karapınar, E., Czerwik, S., Aydi, H.: $(\alpha, \psi)$-Meir-Keeler contraction mappings in generalized $b$-metric spaces. J. Funct. Spaces 2018, Article ID 3264620 (2018)

9. Shatanawi, W.: Fixed and common fixed point for mapping satisfying some nonlinear contraction in $b$-metric spaces. J. Math. Anal. 7(4), 1-12 (2016)

10. Shatanawi, W., Pitea, A., Lazovic, R.: Contraction conditions using comparison functions on $b$-metric spaces. Fixed Point Theory Appl. 2014, 135 (2014). https://doi.org/10.1186/1687-1812-2014-135

11. Roshan, J., Parvaneh, V., Sedghi, S., Shobkolaei, N., Shatanawi, W.: Common fixed points of almost generalized $(\psi, \varphi)_{s}$-contractive mappings in ordered b-metric spaces. Fixed Point Theory Appl. 2013, 159 (2013)

12. Ameer, E., Aydi, H., Arshad, M., De la Sen, M.: Hybrid Ćirić type graphic $(\Upsilon, \Lambda)$-contraction mappings with applications to electric circuit and fractional differential equations. Symmetry 12(3), 467 (2020)

13. Aydi, H., Lakzian, H., Mitrović, Z.D., Radenović, S.: Best proximity points of MF-cyclic contractions with property UC. Numer. Funct. Anal. Optim. 41(7), 871-882 (2020)

14. Parvaneh, V., Haddadi, M.R., Aydi, H.: On best proximity point results for some type of mappings. J. Funct. Spaces 2020, Article ID 6298138 (2020)

15. Aydi, H., Jleli, M., Samet, B.: On positive solutions for a fractional thermostat model with a convex-concave source term via $\psi$-Caputo fractional derivative. Mediterr. J. Math. 17(1), 16 (2020)

16. Kamran, T., Samreen, M., UI Ain, Q.: A generalization of $b$-metric space and some fixed point theorems. Mathematics 5(2), 19 (2017)

17. Aksoy, U., Karapınar, E., Erhan, I.M.: Fixed points of generalized alpha-admissible contractions on $b$-metric spaces with an application to boundary value problems. J. Nonlinear Convex Anal. 17(6), 1095-1108 (2016)

18. Chifu, C., Karapınar, E., Petrusel, G.: Fixed point results in $\varepsilon$-chainable complete $b$-metric spaces. Fixed Point Theory 21(2), 453-464 (2020)

19. Ozyurt, S.G.: On some $\alpha$-admissible contraction mappings on Branciari b-metric spaces. Adv. Theory Nonlinear Anal. Appl. 1(1), 1-13 (2017)

20. Karapınar, E., Fulga, A., Petrusel, A.: On Istratescu type contractions in b-metric spaces. Mathematics $8,388(2020)$

21. Alghamdi, M.A., Gulyaz-Ozyurt, S., Karapınar, E.: A note on extended Z-contraction. Mathematics 8, 195 (2020)

22. Taş, A.: On double controlled metric-like spaces and related fixed point theorems. Adv. Theory Nonlinear Anal. Appl. 5(2), 167-172 (2021)

23. Chifu, $C$ :: Common fixed point results in extended $b$-metric spaces endowed with a directed graph. Results Nonlinear Anal. 2(1), 18-24 (2019) 
24. Mlaiki, N., Aydi, H., Souayah, N., Abdeljawad, T.: Controlled metric type spaces and the related contraction principle. Mathematics 6(10), 194 (2018)

25. Mlaiki, N., Souayah, N., Abdeljawad, T., Aydi, H.: A new extension to the controlled metric type spaces endowed with a graph. Adv. Differ. Equ. 2021, 94 (2021). https://doi.org/10.1186/s13662-021-03252-9

26. Mlaiki, N.: Double controlled metric-like spaces. J. Inequal. Appl. 2020, 189 (2020). https://doi.org/10.1186/s13660-020-02456-z

27. Sintunavarat, W., Cho, Y.J., Kumam, P.: Common fixed point theorems for c-distance in ordered cone metric spaces. Comput. Math. Appl. 62, 1969-1978 (2011). https://doi.org/10.1007/s11784-017-0477-2

28. Mlaiki, N., Aydi, H., Souayah, N., Abdeljawad, T.: An improvement of recent results in controlled metric type spaces. Filomat 34(6), 1853-1862 (2020)

Submit your manuscript to a SpringerOpen ${ }^{\circ}$ journal and benefit from:

- Convenient online submission

- Rigorous peer review

- Open access: articles freely available online

- High visibility within the field

- Retaining the copyright to your article

Submit your next manuscript at $\boldsymbol{\nabla}$ springeropen.com 